\title{
Parameter Setting Problem in the Case of Practical Vehicle Routing Problems with Realistic Constraints
}

\author{
Emir Žunić \\ Info Studio d.o.o. Sarajevo and Faculty of \\ Electrical Engineering, University of \\ Sarajevo, Bosnia and Herzegovina \\ Email: emir.zunic@infostudio.ba
}

\author{
Dženana Đonko \\ Faculty of Electrical Engineering, \\ University of Sarajevo, Bosnia and \\ Herzegovina \\ Email: ddonko@etf.unsa.ba
}

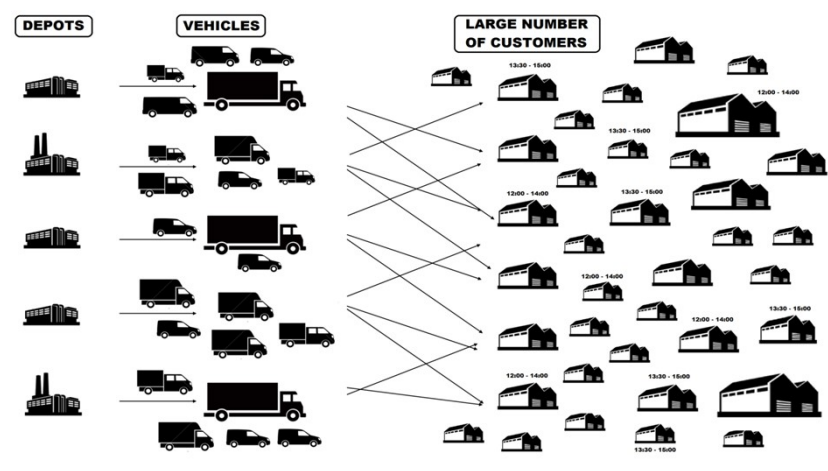

\begin{abstract}
Vehicle Routing Problem (VRP) is the process of selection of the most favorable roads in a road network vehicle should move during the customer service, so as such, it is a generalization of problems of a commercial traveler. Most of the algorithms for successful solution of VRP problems are consisted of several controll parameters and constants, so this paper presents the data-driven prediction model for adjustment of the parameters based on historical data, especially for practical VRP problems with realistic constraints. The approach is consisted of four prediction models and decision making systems for comparing acquired results each of the used models.
\end{abstract}

\section{INTRODUCTION}

$\mathrm{T}$ HE problem of transport route optimization and optimal exploitation of the transport fleet has been explored and constantly improved for a long time. Vehicle routing problem is the name for the entire group of problems requiring the optimal route the transport vehicle or more of them (one vehicle can be used more than once during a single routing) can go around the specific number of customers (delivery points), starting from the central depot and returning there after the customer service. The optimal route is the one with the minimal cost of the road charges [1]. These optimization problems are becoming extremely complex considering a large number of customers. Additionally, those problems become a real challenge considering numerous constraints and facts such as time windows (TW) of customers, time of goods unloading, goods packaging into vehicles, predefined capacity and various vehicles, fixed and variable vehicle costs, etc. These indicators drastically increase the number of available approaches, models and algorithms which could be applied to a complex set of input data. The standard constraints VRP problems differ from are: the number of depots (one or more of them), maximum allowable timing or the length of the vehicle route, different vehicle capacities, customers' demands for delivery or collection of a certain amount of cargo during the service, time windows for beginning and finishing customer service as well as vehicle time windows.
Fig. 1 Rich Vehicle Routing Problem

In a realistic surroundings, it is necessary to take into consideration a huge number of additional constraints, usually being the result of the specificity of the loading and/or unloading locations, specific business processes of the company performing the distribution (collection) or legal decisions and obligations (such as having a rest period for drivers). Such problems are often called Rich Vehicle Routing Problems (RVRP), and one illustrative example is shown on Fig. 1. There are two different approaches in solving RVRP. One of them is the exact algorithms with the aim of finding the optimal solution and proving it to be optimal. Another approach include approximate approaches (heuristic and metaheuristic), with the aim of finding the best possible solution, but without proving it to be optimal. Heuristic approaches and methods are commonly used in complex VRP problems. Neural Networks (NN) as well as Machine Learning (ML) methods have lately been used for the solution of complex VRP problems. Any of these approaches used for solving VRP problems also indicate there are constants and parameters of the algorithm, and their adjustment can make better or worse solution.

The next section presents the basic ways and approaches of adjusting the VRP algorithm parameters. The third section shows the access for adjustment of the control VRP algorithm parameters, consisting of several data transformations. Four algorithms are used for regression. Comparative analysis and discussion of the acquired results are presented in the fourth section. The final section depicts the conclusion of the paper, as well as the guidelines for future researches.

This work was supported by Info Studio d.o.o. Sarajevo 


\section{APPROACHES TO ADJUSTMENT OF VRP ALGORITHM PARAMETERS}

In most of the papers available in literature, there is the fact which points to every realistic VRP problem to be a bit different from its the most similar problem. Two groups of parameters (controlling ones) are the reason of that: (i) Some realistic constraints and constants of the input data, (ii) Constants of the used algorithm. Every company which requires their implementation of the VRP within their surroundings, has its own constraints, defined by the company business polices. Therefore, it is mentioned in literature that the constraints and restrictions in these kinds of problems are non-standard. In the paper [2], Lee describes in details one such problem with the possible solution of the particular example. With the development of modern technology, in the last few years, determination of the parameters based on the available information from GPS and/or GIS system, data on weather condition and forecasts, has also been performed. Several papers deal with the analysis of such systems, and especially interesting papers are [3] those with the data mining methods and techniques for determination of specific realistic constraints of the VRP problems, and [4] those using the predictions of time distances between knots for dynamic routing requirements for emergency vehicles.

One of the most interesting examples of the classical application of realistic and useful data is presented in paper [5], where the concept of data-driven solution of VRP problem is introduced for the first time. The additional phase is also mentioned for the first time in this paper, and it is used in examples that could be applied in realistic surroundings, and that is Human-Computer Interaction Mode (HCIM), which enables the end user to have the ability of manual modification of the suggested routes. No matter how the algorithm for solving VRP problems is considered perfect, there are always realistic situations that are impossible to predict and include in it, so the possibility of manual modification of the suggested routes in practical systems is of a great importance. Each of the analyzed approaches and algorithms for solving VRP problems is composed of specific constants and controlling parameters. Those parameters and constants are used for adjusting specific weight factors, punitive factors according to individual criteria depending on the importance of the very criterion on the final outcome of the realistic situation of vehicle routing, etc. In literature, this approach is defined as the Parameter Setting Problem (PSP). The most interesting paper on this subject was presented by Calvet et al. [6], describing the statistical approach for fine adjusting of the parameters for metaheuristic algorithms, which is also applied to VRP problem.

Analyzing the other available literature dealing with PSP problem, it can be concluded that these problems could be classified into two basic groups [7]: (i) Parameter Control Strategies (PCS), (ii) Parameter Tuning Strategies (PTS). In papers [8]-[9], there is additional sub-group IPTS (Instance-Specific Parameter Tuning Strategies), which includes the characteristics of the instances being applied to. Although there are not many published scientific papers in available literature on the subject of PSP problems, it can be noticed that one of them most interesting applications of it is the improvement of certain segments of VRP, as well as the facilitating of solving VRP problems.

Battiti and Brunato [10] also presented an interesting paper on this subject in which they use the methods of machine learning in combination with statistical methods for fine adjustment of parameters of metaheuristic algorithms. They presented the model that could also be applied for parameters adjustment in other types of algorithms, and one of the most interesting examples is the application for the parameters of neural networks.

Some of the starting ideas for solving realistic VRP problems, and parameters (constants) settings problem are presented in paper [11], where the way of using GPS/GIS data for setting the attribute of the algorithms is presented. In paper [12], the cluster-based analysis and time-series prediction model for reducing the number of traffic accidents is presented.

\section{DATA-DRIVEN APPROACH FOR ADJUSTING THE CONTROL PARAMETERS OF THE VRP ALGORITHMS}

There are three data sets VRP problem is consisted of: depot, vehicles and customers (users). At least one depot must be defined in the problem, and important information that should be collected for the depot is:

- Location: Address, Postal code and place, Geographic position (latitude and longitude)

- Working hours: Opening time, Closing time

If there are more depots distribution is made from, where customers can be served from any other depot, the problem can be modeled as a Multiple Depot Vehicle Routing Problem (MDVRP). But if customers are connected to a particular depot, it is necessary to model more individual VRP problems, for each depot and its buyers individually. In multiple depot problems, vehicles are usually the part of that depot. Basic information about the vehicle include:

- Load space capacity: Capacity [kg], Volume [ $\left.\mathrm{m}^{3}\right]$, Number of pallet positions [pcs], Number of cargo units [pcs]

- Driver's working time

- Departure location

- Arrival location

The capacity of load space is limited by several criteria. For example, in distribution of goods, it is important to pay attention to limited capacity as well as the volume of load space to be sufficient for all the goods being transported. Certain types of goods can be light, but occupy a lot of 
space, so it is necessary to fulfill both constraints. Goods are often put on pallets, so the capacity of vehicle can include the number of pallets possible to place into to load space. The simplest case is in distributing the goods of the same dimensions, so the capacity can be expressed by the maximum number of pieces that could be placed into the load space. Customers' data usually include:

- Location: Address, Postal code and place, Geographic position (latitude and longitude)

- Order: Weight [kg], Volume [ $\left.\mathrm{m}^{3}\right]$, Usage of the pallets [\%], Number of load units [pcs]

- Time limits: The earliest discharge time, The latest discharge time, Estimated discharge duration

Every implemented algorithm solving the realistic VRP problem is consisted of control parameters and constants. The value of these parameters and constants could be adjusted based on historical data, and the model depicting the way of that adjustment is presented in Fig. 2.

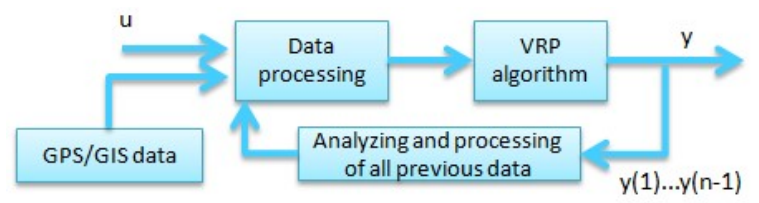

Fig. 2 Approach for adjusting the control parameters based on historical data

The primary goal of realistic VRP problems is to fulfill all constraints. Some of these control parameters are adjusted as described below, using some of the prediction methods and algorithms which will make conclusions based on historical data. On the basis of all the parameters that could have an impact to the final outcome of the solution of VRP problems, several basic ones are sorted out and stored for every route during the testing and production use, since the historical data of practical implementation of VRP algorithms have been used in several largest distribution companies in Bosnia and Herzegovina, dealing with product distribution from their depots to delivery points (shops, supermarkets, etc.). It resulted in creation of a knowledge base being enriched every day, and the mail goal is the adjustment of control parameters and constants on the basis of historical data, which can later become a part of any implemented VRP algorithm. Attributes being sorted out as those affecting the routes are:

- The number of customers

- The number of available vehicles

- The number of available different types of vehicles

- The number of towns

- The total number of restrictions where a customer can't be served by a certain vehicle

- Total number of ordered articles [pcs]

- Total volume of ordered articles $\left[\mathrm{m}^{3}\right]$
- Total weight of all the articles [kg]

- Total duration of TW of all the customers [min]

- Are all the restrictions fulfilled (1: yes, 0: no)

The target attributes affecting the given routes and total cost presenting the control parameters of VRP algorithms in the case of distribution companies are:

\section{- ToleranceWeight \\ - ToleranceVolume \\ - PenaltyDelay \\ - PenaltyCustomersVehicles \\ - CostIncreasing \\ - PenaltyVolumePercentage \\ - PenaltyWeightPercentage}

During the implementation of VRP algorithms, the vehicle can be allowed to be overloaded in weight or volume, for the values of ToleranceWeight and ToleranceVolume. Algorithms for solving VRP problems allow the vehicle to be delayed for customer (to arrive outside of its time window). The violation of this parameter is presented by the PenaltyDelay. If the VRP algorithm is adjusted for solving Site-Dependent Vehicle Routing Problem (SDVRP), the attribute PenaltyCustomersVehicles is used for penalization of rules violations, where the customer can't be served by a particular vehicle. The constant used in increasing the cost, depending on the weight vehicle transports, is presented with the CostIncreasing. The constants penalize reloading of vehicles by weight or volume are PenaltyWeightPercentage and PenaltyVolumePercentage, and they present the cost increasing when the weight/volume of the vehicle is reloaded by $100 \%$. Each of these parameters is determined independently. Firstly, there is a data preprocessing, by excluding only those historical data where all the constraints are fulfilled (value 1 in the column). Then, the removal of redundant attributes was performed by using the Transform Option. Using the Attribute Importance option, the determination of input attribute importance was made for every target attribute. Minimum Descriptor Lenght (MDL) algorithm was used for attribute importance determination. Before that, the normalization of attribute had been performed in a way the volume of the attribute was put to one decimal place.

After all the preprocessings and preparations of input values, the proposed model was created for determing the target attributes. Model for one attribute is shown in Fig. 3.

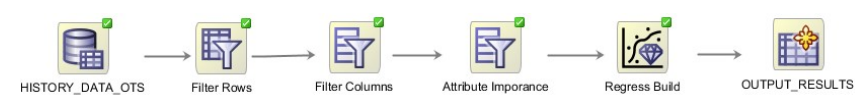

Fig. 3 Prediction model for one attribute

Four regression algorithms were used, and their results are compared: (1) Generalized Linear Models (GLM); (2) Support Vector Machine (SVM); (3) Decision Tree (DT); (4) Naive Bayes (NB). 
The advantage of SVM over other methods is providing better predictions in unseen test data, providing unique optimal solutions for the problem and the existence of less parameter for optimization compared to other methods. The speed of performance is not crucial for the problem wanted to be applied on, so the lack of SVM regression method can be ignored. GLM regression algorithm is chosen because it represents the generalization of the linear regression and is often used in cases where output variables do not have normal distribution. Since the input data point to linear dependence, the GLM choice of the regression algorithm was a logical choice. Basic advantages of a Decision Tree method are: the possibility of generating comprehensive models, relatively small requirements for computer resources (time and memory) and precise importance of some attributes for the specific problem, as well as vast availability of software solution. The lack of Decision Trees is their instability, because small fluctuations in data sample can result in huge variations in assigned classifications. The advantage of the Naive Bayes classifier is its robustness to errors obtained in data collecting or missing attribute value in training session. Errors do not have a lot of impact on probabilities since they are of average value, while missing data are simply ignored during the calculating of probability. Also, the Naive Bayes classifier is robust to irrelevant attributes, too.

\section{RESULTS DISCUSSION}

As previously mentioned, for each of the control parameters, the independent model was created with four regression algorithms: GLM, SVM, Decision Tree and Naive Bayes algorithm. After the results were obtained, for each of the parameters, the Decision Support System (DSS) was created which, on the basis of regression results, chose the predicted value of the algorithm that had higher Predictive Confidence. In order to enrich the control parameters knowledge base, VRP productive algorithms were run for more than 10.000 times, with all the constraints fulfilled for many different days and input parameters. Data with testing and validating of prediction models are provided at the 4TU Research Data Center [13], to be available to other researchers for their works and eventual comparison of results. For each of the control parameters, there was a result comparison of confidence/accuracy - Predictive Confidence [\%], which was presented for each of the input data set in Table I.

As seen from the presented results, it is not difficult to conclude that the SVM always provided better predictive results for each of the control parameters compared to GLM algorithm (Table I - left), while the prediction models based on the Decision Tree and Naive Bayes algorithms, always showed much worse results (Table I - right), and therefore, the Decision Support System (DSS) preferred the prediction results of the SVM algorithm. Analyzing the Table I in details, it is easy to conclude that the lack of Decision Tree is their instability, because small fluctuations may result in large variations in the assigned classifications, which was the case. The lack of the Naive Bayes prediction algorithm is presumably the independence of attributes, which make this classifier delicate to correlated attributes. Attributes in strong correlation can degrade performances of the classifiers, which can be solved by removing certain attributes, which is also the case in this example.

$\begin{aligned} & \text { TABLE I - COMPARATIVE RESUlTS OF USED REGRESSION GLM, SVM, } \\
& \text { DT AND NB MODELS - PREDICTIVE CONFIDENCE [\%] }\end{aligned}$
\begin{tabular}{ccccc} 
Parameter & GLM & SVM & DT & NB \\
\hline ToleranceWeight & 91.766 & 96.123 & 81.336 & 84.323 \\
ToleranceVolume & 81.995 & 90.201 & 79.928 & 80.905 \\
PenaltyDelay & 84.956 & 89.551 & 81.555 & 82.007 \\
PenaltyCustomersVehicles & 92.031 & 96.439 & 82.309 & 85.314 \\
CostIncreasing & 81.276 & 89.996 & 78.998 & 80.229 \\
PenaltyVolumePercentage & 89.133 & 91.853 & 83.892 & 84.934 \\
PenaltyWeightPercentage & 90.006 & 92.698 & 84.801 & 84.956 \\
\hline
\end{tabular}

Implemented Attribute Importance segment at the target values in the prediction model enables to determine the importance of each of the input attributes on the target control parameter. The average importance of the input parameters on the input control variables, as well as their order, is shown in Table II. Analyzing the average value of input attribute influences for each of the control parameters, it is concluded that the input parameters affect the output prediction control parameters in that order shown in Table II. Such results were expected because routings made on realistic data were extremely complex with strict constraints, while the number of available vehicles was very small. The fact is that out of 8 available vehicles for routing, seven of them were of different type, which significantly affects the result and complexity of algorithm implementation. It is easy to conclude that these parameters are the most important for adjusting the algorithm control parameters. Also, the customers' time windows are significant for the control parameters, which affect the complexity of finding a solution.

TABLE II - ATTRIBUTE IMPORTANCE RESULTS

\begin{tabular}{ccc}
\hline $\begin{array}{c}\text { Input Parameter } \\
\text { vehicles }\end{array}$ & $\begin{array}{c}\text { Importance } \\
\text { Number }\end{array}$ & $\begin{array}{c}\text { Importance } \\
\text { Weight }\end{array}$ \\
\hline The number of available different types of & 1 & 0.871 \\
The number of available vehicles & 2 & 0.861 \\
Total duration of time windows of all the & 3 & 0.796 \\
customers [min] & 4 & 0.758 \\
Total number of ordered articles [pcs] & 5 & 0.747 \\
The number of customers & 6 & 0.741 \\
Total volume of ordered articles [ $\left.{ }^{3}\right]$ & 7 & 0.727 \\
The number of towns & 8 & 0.582 \\
Total weight of all the articles [kg] & & 0.303 \\
The total number of restrictions where a & 9 & \\
customer can't be served by a certain \\
vehicle
\end{tabular}

According to Table II, the parameter with the least significance for adjusting the value of control parameters is the number of constraints the customer can't be served by a vehicle. The very number is presented in the form of the summary indicator, but if it were presented in terms of ratio 
of the customer and the number of vehicles that could serve him, the significance of that parameter would certainly be much greater, even the most significant. For each of the control parameters, the results are graphically displayed, which is example of the ToleranceWeight parameter shown in Fig, 4 (left). The important indicator in these analyses is the Mean Absolute Error (MAE) prediction. Results of the MAE for the example of ToleranceWeight parameter are shown in Fig. 4 (right).

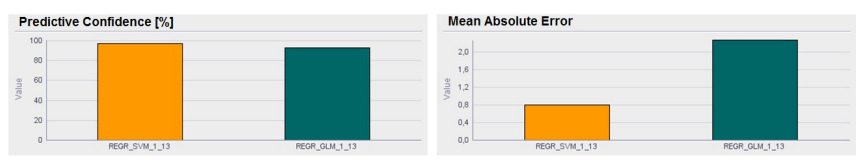

Fig. 4 Predictive confidence [\%] and Mean Absolute Error results: SVM and GLM

It is also possible to observe the comparison of Residual (Residual is the difference between expected and predicted value of the dependent variable) for each of the control parameters. The example of the comparison of the ToleranceWeight parameter is shown in Fig. 5.
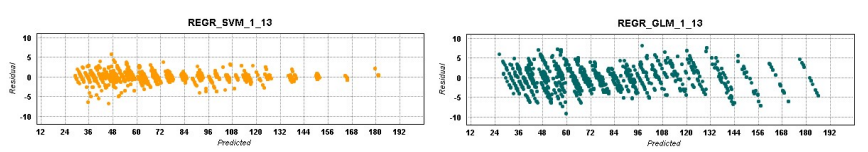

Fig. 5 Residual comparisons: SVM (left) and GLM (right)

Figure 6 shows that for the each attribute except the Predictive Confidence indicator, it is possible to obtain many other parameters (primarily refers to the prediction value errors), which enables to make comparisons and select the model which satisfies the needs and expectations more.

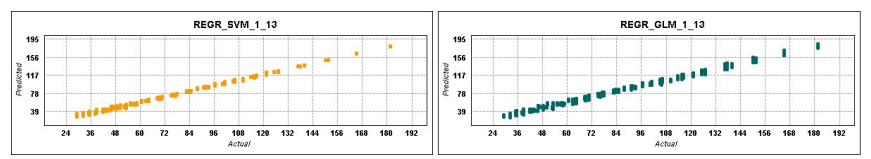

Fig. 6 Comparison of realistic and predicted values - SVM and GLM

It is also simple to make comparison of the actual and predicted value, and make the same conclusion that the SVM algorithm presented much better results compared to the remaining 3 prediction algorithms already used. Thus, every analysis points to the fact that the SVM algorithm is much more superior than other algorithms already used, as well as methods for the purpose of adjusting the control parameters of the practically applicable algorithms for solving the complex VRP problems.

\section{CONCLUSION}

Regardless of what approach (exact or heuristics) is used for successful solving the VRP problem, most of the proposed algorithms are consisted of constants in control parameters whose adjustment give better or worse solutions. This paper presents the innovative approach of adjustment of these parameters on the basis of available historical data by using the prediction models. Four of the prediction models were used, where SVM algorithm proved to have much better and more superior results for all the tests compared to other prediction models. For each of the analyzed control parameters in the case of SVM model, the predictive accuracy was over $90 \%$. The advantage of SVM over other methods being used is providing better predictions in unseen test data, providing unique optimal solutions for the training problem, and the existence of less optimization parameters compared to other methods. The execution speed is not crucial for the problem, so the lack of SVM regression method can be neglected in this case.

Guidelines for the future researches in this area would include the application of neural networks for determination of the values of the VRP algorithm control parameters, or even the prediction based on time series. Surely, the progress should be realized by even more input set of realistic data in this segment of the proposed approach.

\section{REFERENCES}

[1] Dantzig, G. B., Ramser, J. H. 1959. The truck dispatching problem. Management science. 6(1):80-91, https://doi.org/10.1287/mnsc.6.1.80

[2] Lee, W. L. 2013. Real-Life Vehicle Routing with Non-Standard Constraints. Proceedings of the World Congress on Engineering (WCE). I:432-437

[3] Hu, X., Huang, M., Zeng, A. 2007. An intelligent solution system for a vehicle routing problem in urban distribution. International Journal of Innovative Computing, Information and Control. 3:189-198

[4] Musolino, G., Rindone, C., Polimeni, A., Vitetta, A. 2013. Travel Time Forecasting and Dynamic Routes Design for Emergency Vehicles. Procedia - Social and Behavioral Sciences. 87:193-202, https://doi.org/10.1016/j.sbspro.2013.10.603

[5] Fu, C., Wang, H. 2010. The solving strategy for the real-world vehicle routing problem. 3rd International Congress on Image and Signal Processing. 3182-3185, https://doi.org/10.1109/CISP.2010.5647968

[6] Calvet, L., Juan, A. A., Serrat, C., Ries, J. 2016. A statistical learning based approach for parameter fine-tuning of metaheuristics. SORT Statistics and Operations Research Transactions. 40(1):201-240

[7] Birattari, M., Kacprzyk, J. 2009. Tuning metaheuristics: A machine learning perspective. Springer, Vol. 197. ISBN: 3642004822 9783642004827

[8] Montero, E., Riff, M. C., Neveu, B. 2014. A beginner's guide to tuning methods. Applied Soft Computing. 17:39-51, https://doi.org/10.1016/j.asoc.2013.12.017

[9] Ries, J., Beullens, P., Salt, D. 2012. Instance-specific multi-objective parameter tuning based on fuzzy logic. EJOR. Elsevier. 218:305-315, https://doi.org/10.1016/j.ejor.2011.10.024

[10] Battiti, R., Brunato, M. 2010. Reactive Search Optimization: Learning While Optimizing. Handbook of Metaheuristics. International Series in Operations Research \& Management Science. 543-571, https://doi.org/10.1007/978-1-4419-1665-5 18

[11] Žunić, E., Hindija, H., Beširević, A., Hodžić, K., Delalić, S. 2018. Improving Performance of Vehicle Routing Algorithms using GPS Data. 14th Symposium on Neural Networks and Applications (NEUREL). 1-4. https://doi.org/10.1109/neurel.2018.8586982

[12] Žunić, E., Djedović, A., Đonko, D. 2017. Cluster-based analysis and time-series prediction model for reducing the number of traffic accidents. International Symposium ELMAR. 25-29, https://doi.org/10.23919/ELMAR.2017.8124427

[13] Žunić, E. (Emir). 2018. Real-world VRP data with realistic nonstandard constraints - parameter setting problem regression input data. 4TU.Centre for Research Data. Dataset. Available at: https://doi.org/10.4121/uuid:97006624-d6a3-4a29-bffae8daf60699d8 